\title{
Magnetic Field Landscapes Guiding the Chemisorption of Diamagnetic Molecules
}

By Florian Ahrend, Ulrich Glebe, Líney Árnadóttir, Joe E. Baio, Daniel A. Fischer, Cherno Jaye, Bonnie O. Leung, Adam P. Hitchcock, Tobias Weidner, Ulrich Siemeling*, and Arno Ehresmann*

\section{Supporting Information.}

\section{Content:}

Section 1: $\quad$ Fabrication of artificial strayfield landscapes

Section 2: $\quad$ Synthesis, molecule description and preparation of submonolayers

Section 3: Time-of-flight secondary ion mass spectrometry (ToF-SIMS)

Section 4: $\quad$ X-ray photoemission electron microscopy (X-PEEM)

Section 5: $\quad$ Near-edge X-ray absorption fine structure (NEXAFS) imaging

Section 1: $\quad$ Fabrication of artificial strayfield landscapes

$\mathrm{Si} / \mathrm{Cu}^{50 \mathrm{~nm}} / \mathrm{Ir}_{17} \mathrm{Mn}_{83}{ }^{10 \mathrm{~nm}} / \mathrm{Co}_{70} \mathrm{Fe}_{30}{ }^{5 \mathrm{~nm}} / \mathrm{Au}^{5-8 \mathrm{~nm}}$ exchange-bias layer systems were deposited by DC magnetron sputtering in a Leybold-Hereaus $\mathrm{Z} 400$ at a base pressure of about $1 \cdot 10^{-6}$ mbar. After layer deposition the exchange-bias effect was initiated by field cooling at $300^{\circ} \mathrm{C}$ for $1 \mathrm{~h}$ at about $5 \cdot 10^{-6}$ mbar in an external magnetic field of 1000 Oe. The resulting in-plane exchangebias field was $\mathrm{H}_{\mathrm{eb}, \mathrm{FC}}=-450$ Oe as characterized by vibrating sample magnetometry (VSM). Subsequently, UV-lithography was used to fabricate a resist mask consisting of parallel stripes, alternating covered and uncovered by photoresist (AZ-1505 from MicroChemicals®). Two different periodicities of stripe shaped masks were used, as explained in the main text. The samples were then magnetically patterned by $10 \mathrm{keV} \mathrm{He}{ }^{+}$IBMP, bombarding the layer systems with resist mask by $1 \cdot 10^{15}$ ions $\left(\mathrm{cm}^{-2}\right)$ of $10 \mathrm{keV} \mathrm{He}^{+}$ions from a home-built plasma ion source $^{37}$ in an applied bombardment field of 900 Oe directed antiparallel to the initialization field of the field cooling step. After resist removal (4 min in $3 \%$ aqueous $\mathrm{KOH}$ in ultra sonic followed by acetone and isopropanol rinsing) the resulting magnetic patterns are alternating wider and narrower parallel stripes with in-plane head-to-head/ tail-to-tail magnetization configurations perpendicular to their long stripe axes in adjacent domains (see scheme 1). Narrower and wider stripes were fabricated in order to identify in the later characterization experiments bombarded 
and nonbombarded areas and to fabricate areas with higher magnetic flux density (the narrow stripes) and lower magnetic flux density (the wider stripes). Characterization measurements by VSM show a double hysteresis with two shifted loops, one shifted by the initial $\mathrm{H}_{\mathrm{eb}, \mathrm{FC}}=-450$ Oe and the other shifted by $\mathrm{H}_{\mathrm{eb}, \mathrm{IB}}=300 \mathrm{Oe}$, corresponding to areas with ion bombardment modified exchange-bias. The fabricated head-to-head/ tail-to-tail magnetization pattern results in maximum magnetic charges at the domain walls ${ }^{19}$ and therefore to maximum magnetic stray fields above the sample surface.

Section 2: $\quad$ Synthesis, molecule description and preparation of submonolayers

The used chemical compounds are peripherally alkylthio substituted since this increases their solubility in common organic solvents and enables a multipoint attachment on gold surfaces through sulfur gold bonds. [BClSubpc' $\left(\mathrm{S} n-\mathrm{C}_{12} \mathrm{H}_{25}\right)_{6}$ ] (scheme 1) was synthesized following the route for its $n$-octylthio derivative. ${ }^{39}$ These diamagnetic molecules are known to form stable selfassembled monolayers on pure (unpatterned) gold layers. ${ }^{20}$ The deposition of the adsorbate onto the magnetically patterned sample surfaces was performed from solution. Solutions with a concentration of $10 \mu \mathrm{M}$ (for the X-PEEM characterization measurements) or $1 \mu \mathrm{M}$ (for ToFSIMS and NEXAFS imaging), respectively, in $\mathrm{CH}_{2} \mathrm{Cl}_{2}$ were used. The samples were immersed into the solution for $1 \mathrm{~min}$ at room temperature, followed by $12 \mathrm{~h}$ in pure solvent. After immersion, the samples were sonicated and carefully rinsed with copious amounts of dichloromethane in order to remove only slightly bound molecules, blown dry with nitrogen, and kept under nitrogen atmosphere until they were characterized.

\section{Section 3: $\quad$ Time-of-flight secondary ion mass spectrometry (ToF-SIMS)}

ToF-SIMS data were collected on an ION-TOF 5-100 instrument using a $\mathrm{Bi}_{3}{ }^{++}$primary ion source. Positive and negative spectra were acquired with a pulsed primary ion beam $(25 \mathrm{keV}, 0.3$ pA) in high-current bunched mode under static conditions (primary ion dose $<10^{11}$ ions $\mathrm{cm}^{-2}$ ). The mass-resolution $(\mathrm{m} / \Delta \mathrm{m})$ of the negative secondary ion spectra was typically about 5000 for the $\mathrm{m} / \mathrm{z}=25$ peak and about 6000 for the $\mathrm{m} / \mathrm{z}=27$ peak in the positive spectra. Positive ion spectra were mass-calibrated using the $\mathrm{CH}_{2}{ }^{+}, \mathrm{C}_{2} \mathrm{H}_{2}{ }^{+}, \mathrm{C}_{3} \mathrm{H}_{4}{ }^{+}, \mathrm{C}_{4} \mathrm{H}_{6}{ }^{+}$, and $\mathrm{C}_{6} \mathrm{H}_{11}{ }^{+}$peaks and the negative ion mass spectra were mass-calibrated using the $\mathrm{CH}^{-}, \mathrm{OH}^{-}, \mathrm{C}_{3} \mathrm{H}^{-}$, and $\mathrm{C}_{4} \mathrm{H}^{-}$peaks. Whenever possible, peak identities were confirmed using the natural isotopic ratio of the elements.

\section{Section 4: $\quad$ X-ray photoemission electron microscopy (X-PEEM)}

X-PEEM measurements were carried out at the Advanced Light Source (ALS) on bending magnet beamline 7.3.1. ${ }^{40}$ For $\mathrm{C} 1 \mathrm{~s}$ spectra, a $100 \mathrm{~nm}$ titanium filter was used to eliminate second order light. This filter was removed during the collection of Fe XMCD spectra. A shutter with a 
$0.1 \mathrm{~s}$ response time was used to block the photon beam during the time required for the images to be transferred from the CCD camera to the computer and to step the energy in order to minimize radiation damage. X-PEEM data analysis was performed with the aXis2000 software package. ${ }^{41}$ For the C 1s stack, right handed circularly polarized light was used, comparison with left handed circularly polarized light showed no difference between the two C1s stacks. The spectra were extracted and normalized such that the pre-edge and post-edge NEXAFS spectra overlapped. A suitable $I_{o}$ could not be obtained, thus, the spectra below are shown without $I_{0}$ correction. The $\mathrm{C} 1 \mathrm{~s}$ spectra were externally calibrated with a polystyrene sample, assigning the $\mathrm{C} 1 \mathrm{~s} \rightarrow \pi^{*}$ transition to $285.15 \mathrm{eV}$.

Figure S1 shows results of the X-PEEM measurement in grey scale with extracted NEXAFS spectra from the areas depicted in (c).
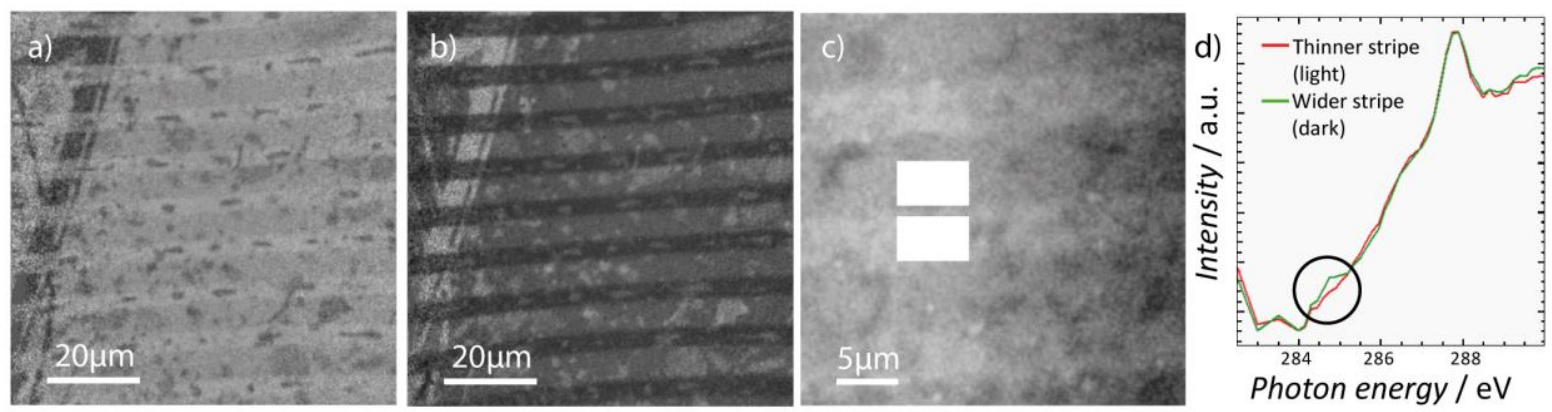

Figure S1. X-PEEM images of series 2 sample. a) Signal from Fe $2 p \rightarrow 3 d$ absorption by left handed circularly polarized light after normalization $\left(\mathrm{L}_{3}\right.$ edge/ $\mathrm{L}_{2}$ edge). (b) The same as (a) but with right handed circularly polarized light. (c) Image obtained at the $\mathrm{C} 1 \mathrm{~s} \rightarrow \pi^{*}$ emission absorption, white boxes denote the identical areas where the $\mathrm{C}$ 1s NEXAFS spectra were extracted from the stack. (d) Normalized NEXAFS spectra obtained from the white boxes in (a). The black circle highlights the $\mathrm{C}=\mathrm{C} \pi^{*}$ transition at $285.15 \mathrm{eV}$ and reveals the presence of more subphthalocyaninatoboron molecules on the wider dark stripes as compared to the lighter white stripes.

Section 5: $\quad$ Imaging near-edge X-ray absorption fine structure (NEXAFS)

NEXAFS images were collected on the parallel imaging system at the NIST U7A beamline at the National Synchrotron Light Source (NSLS - Brookhaven National Laboratory) using a large area rapid imaging analytical tool (LARIAT, Synchrotron Research Inc.). X-rays, with energy scanned around the carbon $K$-edge (270-340 eV), were rastered across an $18 \times 13 \mathrm{~mm}^{2}$ area on the sample. Carbon $K$-edge scans were performed with a step size of $0.1 \mathrm{eV}$ and a dwell time of $2 \mathrm{~s}$. The emitted photoelectrons were extracted by a full field imaging parallel magnetic field and guided to an electron yield detector. An entrance grid bias located in front of the electron yield detector was set to $50 \mathrm{~V}$ to enhance surface sensitivity. This procedure yields a series of two- 
dimensional NEXAFS images with a $50 \mu \mathrm{m}$ spatial resolution. To compensate for the effect of incident beam intensity fluctuations and absorption features in the beamline optics, the PEY signals were normalized by the electron yield of a clean gold mesh located upstream along the path of the incident X-ray beam. This NEXAFS imaging endstation can handle a range of conducting and insulating samples without charging effects. ${ }^{35,36}$ All images presented in this study have been pre- and post-edge normalized.

This material is available free of charge via the Internet at http://pubs.acs.org. 